\title{
Eosinophil granuloma due to parasite treated by laparoscopic and endoscopic cooperative surgery: a case report
}

\author{
Toshikatsu Tsuji ${ }^{* *}$ and Noriyuki Inaki
}

\begin{abstract}
Background: Gastric eosinophilic granuloma caused by parasitic infection is rare. It is often suspected to be a malignant disease and it is difficult to diagnose. We successfully diagnosed and removed a gastric eosinophilic granuloma using laparoscopic and endoscopic cooperative surgery (LECS).
\end{abstract}

Case presentation: A 35-year-old woman visited our hospital because of epigastric pain. Upper gastrointestinal endoscopy revealed a $15 \mathrm{~mm}$ submucosal tumor (SMT) with changes in the folds, such as enlargement and convergence, located in the greater curvature of the lower gastric body. Computed tomography (CT) showed a dense, nonenhanced area of $15 \mathrm{~mm}$ at the same site. SMT was suspected, but undifferentiated cancer could not be excluded. We performed laparoscopic partial gastrectomy using LECS for resection biopsy.

Histopathological examination showed an SMT $8 \times 8 \times 5 \mathrm{~mm}$ in size with an unclear boundary and necrosed insects at the core of the tumor. There was marked eosinophilic infiltration around the area. The diagnosis was gastric granuloma caused by parasitic infection.

Conclusions: It is difficult to differentiate gastric eosinophilic granuloma caused by parasitic infection from malignant disease. In this case, LECS is considered a minimally invasive and useful procedure.

Keywords: Gastric eosinophilic granuloma, Parasitic infection, Anisakiasis, Laparoscopic and endoscopic cooperative surgery (LECS)

\section{Background}

Anisakiasis is a parasitic disease caused by the consumption of raw fish infected with Anisakis. This disease has been reported in Japan, Korea, the Netherlands, and western Europe, where raw fish is eaten. Gastric anisakiasis is divided into two types: the acute type (acute anisakiasis) and the chronic type (eosinophilic granuloma). The acute type, which develops sudden severe abdominal pain, is well known. The chronic type is relatively rare, accounting for $2 \sim 4 \%$ of anisakiasis cases. It is difficult to differentiate the chronic type of anisakiasis from malignant disease by endoscopy and imaging to achieve a definitive diagnosis before surgery. We performed laparoscopic and endoscopic cooperative surgery (LECS)

\footnotetext{
*Correspondence: toshi_toshi25@yahoo.co.jp

${ }^{1}$ Department of Gastroenterological Surgery, Ishikawa Prefectural Central

Hospital, 2-1 Kuratsukihigashi, Kanazawa, Ishikawa 9208530, Japan

Full list of author information is available at the end of the article
}

for therapeutic diagnosis, which is considered a minimally invasive and useful procedure in this disease.

\section{Case presentation}

A 35-year-old woman visited our hospital because of epigastric pain. Laboratory data showed white blood cell count of $5.83 \times 10^{3} / \mu \mathrm{L}$, eosinophils $3.8 \%$. Upper gastrointestinal endoscopy revealed a $15 \mathrm{~mm}$ SMT with changes in the folds, such as enlargement and convergence, located in the greater curvature of the lower gastric body (Fig. 1). The mucosa was partially erosive, and the tumor was elastic and hard. Endoscopic ultrasound (EUS) findings revealed no regions that could be identified as a tumor at either $12 \mathrm{MHz}$ or $20 \mathrm{MHz}$, and the layered structure was maintained. CT showed a localized nonenhanced nodule $15 \mathrm{~mm}$ in size at the same site (Fig. 2). Thus, gastric SMT was suspected, but undifferentiated cancer could not be excluded. Biopsy was performed several times, and deep biopsy was also performed, but no malignant findings 


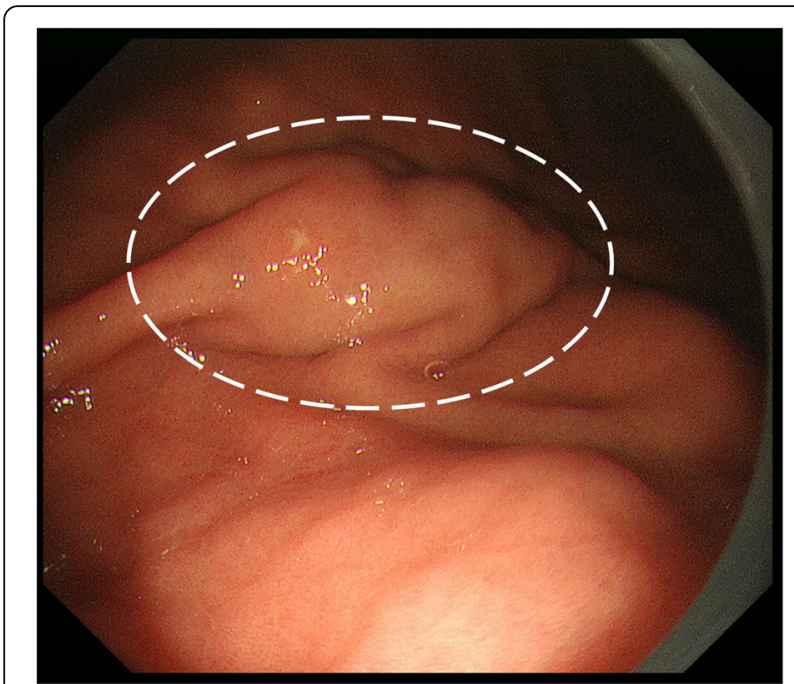

Fig. 1 Upper gastrointestinal endoscopy findings: $15 \mathrm{~mm}$ SMT with the changes in the fold such as enlargement and convergence located in the greater curvature of the lower gastric body. The mucosa was partially erosive

were observed. We planned to carry out total biopsy after obtaining informed consent because malignant disease could not be excluded.

We performed laparoscopic partial gastrectomy via LECS. The port arrangement was shifted to the foot side relative to the port arrangement in conventional gastrectomy because of severe gastroptosis. Five ports (one 12-mm, one 5$\mathrm{mm}$, and three $2-\mathrm{mm}$ ports) were placed. The tumor location was confirmed in the greater curvature of the lower gastric body by endoscopy. The omentum around the tumor was minimally removed. A mucosal incision was made around the tumor with an adequate margin. The most anal side of the mucosal incision was endoscopically perforated. The rest of the specimen was circumferentially resected using laparoscopic coagulating shears (Fig. 3). The specimen was stored in a plastic bag and orally extracted.

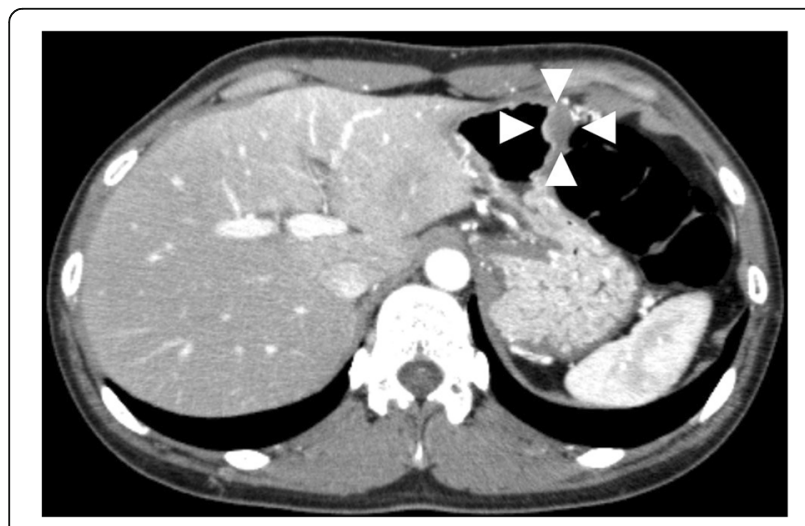

Fig. 2 Enhanced abdominal $C T$ findings. The dense stain defective area of $15 \mathrm{~mm}$ at the greater curvature of the gastric body
The defect in the stomach wall was closed with continuous full-layer suturing using barbed sutures (3-0 V-LOC ${ }^{\mathrm{TM}} 180$, Medtronic plc, Dublin, Ireland).

The patient was discharged on the seventh postoperative day without complications. At the 6-month postoperative checkup, she had no complaints, such as bloating, pyrosis, and burp.

Histopathological examination showed a yellowish white SMT $8 \times 8 \times 5 \mathrm{~mm}$ in size with an unclear boundary, and necrosed insects were found at the core of the tumor. There was marked eosinophilic infiltration around the area. The final diagnosis was gastric granuloma caused by parasitic infection (Fig. 4).

\section{Discussion}

In our case, therapeutic diagnosis was less invasively performed by LECS for the granulomatous tumor which is difficult to differentiate as malignant tumor. The histopathological findings revealed that eosinophilic granuloma formation due to a chronic foreign body reaction. In this kind of chronic type, an EUS usually reveals a central hyperechoic area, which is a characteristic finding of Anisakis granuloma [1]. It also represents a circular or elliptical structure with hyperechoic signals in the center of a hypoechoic mass with an unclear boundary.

However, these typical findings could not be confirmed in our case. Its endoscopic finding revealed a somewhat malignant tumor (e.g., gastrointestinal stromal tumor (GIST), metastatic gastric tumor) with ulcer formation and changes in the folds, such as enlargement, convergence, and erosion, on its top [2]. Thus, we ultimately suspected malignancy; biopsy was performed several times, which resulted in failure to diagnose. Although we considered gastrectomy with lymphadenectomy in case of malignant tumor, we finally decided to perform LECS for the therapeutic total biopsy. As a result, we could obtain a definitive diagnosis without complications and patient could be treated less invasively preserving almost the whole stomach.

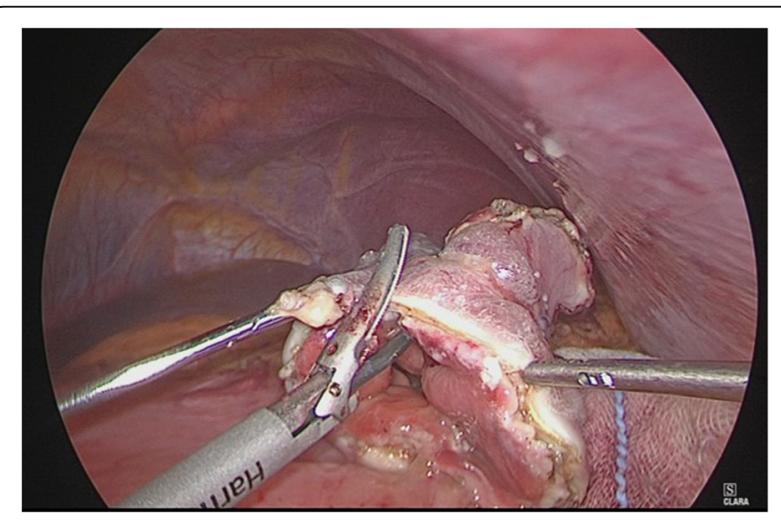

Fig. 3 Operative findings. After mucosal incision, the specimen was circumferentially resected by using laparoscopic coagulating shears 


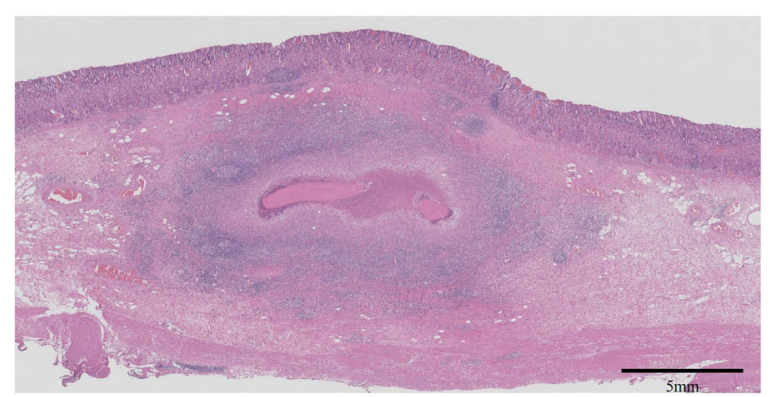

Fig. 4 Histopathological findings. $8 \times 8 \times 5$ mm submucosal tumor with unclear boundary was observed and necrosed insect was found as the core of the tumor. There was marked eosinophil infiltration around the area

LECS is a surgical technique for SMT removal reported by Hiki et al. and a procedure that can minimize deformation and dysfunction by avoiding excessive resection of the stomach wall. However, when obvious ulcers are involved, it is necessary to prevent the spread of the tumor cells, and it may be better to consider the following methods that do not expose the tumor to the abdominal cavity: inverted LECS, nonexposed endoscopic wall inversion surgery (NEWS), and a combination of laparoscopic and endoscopic approaches to neoplasia with a nonexposure technique (CLEAN-NET) [3-7].

We chose original LECS procedure and perforated gastric wall during resection of the tumor; however, we carefully paid attention not to spoil the gastric juice out from the stomach and to let the surface of the tumor touch to the abdominal cavity. The diagnosis was gastric granuloma caused by parasitic infection, excluding malignancy or typical SMT such as GIST and leiomyoma. LECS was a valid procedure for this case as a result.

The patient did not eat raw fish 1 week before she visited at least. However, she usually eats raw fish. It is also important to keep in mind the gastric eosinophilic granuloma caused by parasitic infection, especially in regions where raw fish is eaten.

\section{Conclusions}

LECS is a favorable less invasive candidate procedure for therapeutic diagnosis when we encounter the granuloma which is difficult to diagnose.

\footnotetext{
Abbreviations

CLEAN-NET: Combination of laparoscopic and endoscopic approaches to neoplasia with a nonexposure technique; CT: Computed tomography; EUS: Enhanced ultrasound; GIST: Gastrointestinal stromal tumor; LECS: Laparoscopic and endoscopic cooperative surgery; NEWS: Nonexposed endoscopic wall inversion surgery; SMT: Submucosal tumor
}

Authors' contributions

$\Pi$ is the first author and prepared the manuscript under the supervision of $\mathrm{NI}$. TT and NI performed the surgery. Both authors read and approved the final manuscript.

\section{Funding}

The authors declare that they received no funding support for this report.

\section{Availability of data and materials}

Data sharing is not applicable to this article, because no datasets were generated or analyzed during this study.

\section{Ethics approval and consent to participate}

Not applicable

\section{Consent for publication}

Consent to publish was obtained from this patient and the identity of this patient was protected.

\section{Competing interests}

The authors declare that they have no competing interests.

\section{Author details}

'Department of Gastroenterological Surgery, Ishikawa Prefectural Central Hospital, 2-1 Kuratsukihigashi, Kanazawa, Ishikawa 9208530, Japan.

${ }^{2}$ Department of Surgery, Juntendo Urayasu Hospital, Juntendo University, 2-1-1, Tomioka, Urayasu-shi, Chiba 2790021, Japan.

Received: 10 October 2019 Accepted: 31 December 2019

Published online: 10 January 2020

\section{References}

1. Sasaki K, Miyachi K, Aoki H, et al. Endoscopic ultrasonography of the granuloma in gastric anisakiasis. Gastroenterological Endoscopy. 2002;44: 996-1000.

2. Kang DB, Park WC, Lee JK. Chronic gastric anisakiasis provoking a bleeding gastric ulcer. Ann Surg Treat Res. 2014;86:270-3.

3. Nunobe S, Hiki N, Gotoda T, et al. Successful application of laparoscopic endoscopic cooperative surgery(LECS)for a lateral-spreading mucosal gastric cancer. Gastric Cancer. 2012;15:338-42.

4. Hiki N, Nunobe S, Matsuda T, et al. Laparoscopic endoscopic cooperative surgery. Dig Endosc. 2015;27:197-204.

5. Mitsui T, Niimi $\mathrm{K}$, Yamashita H, et al. Non-exposed endoscopic wall-inversion surgery as a novel partial gastrectomy technique. Gastric Cancer. 2014;17: 594-9.

6. Goto O, Takeuchi H, Kawakubo H, et al. First case of non-exposed endoscopic wall-inversion surgery with sentinel node basin dissection for gastric cancer. Gastric Cancer. 2015;18:434-9.

7. Inoue H, Ikeda H, Hosoya T, et al. Endoscopic mucosal resection, endoscopic submucosal dissection, and beyond: full-layer resection for gastric cancer with nonexposure technique(CLEAN-NET). Surg Oncol Clin N Am. 2012;21: $129-40$.

\section{Publisher's Note}

Springer Nature remains neutral with regard to jurisdictional claims in published maps and institutional affiliations. 